\title{
Upregulation of LINC00511 expression by DNA hypomethylation promotes the progression of breast cancer
}

\author{
Chunxiao Liu ${ }^{1}$, Yuting Xu ${ }^{1}, \mathrm{Xu}$ Liu $^{1}$, Yingqiang Fu ${ }^{1}$, Kaiyuan Zhu ${ }^{1}$, Zhenbo Niu ${ }^{1}$, Jiaxin Liu ${ }^{1}$, Cheng Qian ${ }^{1,2} \wedge$ \\ ${ }^{1}$ Department of Breast Surgery, Harbin Medical University Cancer Hospital, Harbin, China; ${ }^{2}$ Translational Medicine Research and Cooperation \\ Center of Northern China, Heilongjiang Academy of Medical Sciences, Harbin, China \\ Contributions: (I) Conception and design: C Liu; (II) Administrative support: C Qian; (III) Provision of study materials or patients: Y Fu, K Zhu, Z \\ Niu; (IV) Collection and assembly of data: C Liu, Y Xu, J Liu; (V) Data analysis and interpretation: C Liu, Y Xu, X Liu; (VI) Manuscript writing: All \\ authors; (VII) Final approval of manuscript: All authors. \\ Correspondence to: Cheng Qian, MD, PhD. Department of Breast Surgery, Harbin Medical University Cancer Hospital, 150 Haping Road, Nangang, \\ Harbin, China. Email: qiancheng@ems.hrbmu.edu.cn.
}

Background LINC00511 is a newly discovered long intergenic nonprotein-coding RNA (Ribonucleic acid) with unknown.

Method: Differential gene expression analysis was performed on breast cancer microarray data, and the upregulated expression of LINC00511 in breast cancer tissues and breast cancer cell lines was verified by qRT-PCR (quantitative Reverse Transcription-Polymerase Chain Reaction). A cohort study revealed a correlation between the expression of LINC00511 and the clinicopathological features in breast cancer patients. The effects of LINC00511 on breast cancer migration and invasion were studied in vitro. Then, an experiment using the Illumina Infinium Human Methylation450 Beadchip data was conducted to study the role of DNA (Deoxyribonucleic acid) methylation in LINC00511 expression, and DAVID (Database for Annotation, Visualization and Integrated Discovery) Functional Annotation Bioinformatics Microarray Analysis was used to determine the biological functions and potential pathways of LINC00511 in breast cancer. Then, LINC00511 and key genes associated with breast cancer disease progression were further studied in TCGA (The Cancer Genome Atlas), and western blotting was used to verify the results at the protein level. Finally, we further studied the effect of LINC00511 on Panobinostat drug sensitivity in breast cancer and its effect on the prognosis of breast cancer patients.

Results: LINC00511 was upregulated in breast cancer patients. The expression of LINC00511 was closely related to lymph node metastasis, tumor size and molecular subtypes of breast cancer. The in vitro studies revealed that LINC00511 could promote the migration and invasion in MDA-MB-231 and MCF-7 cells. In terms of mechanism, DNA hypomethylation promoted the expression of LINC00511, furthermore LINC00511 promoted the expression of Wnt10A, E2F2, TGFA, and MET, which participate in the progression of breast cancer. In addition, LINC00511 reduced the sensitivity of breast cancer cells to Panobinostat. Moreover, breast cancer patients with a high expression of LINC00511 had a poor prognosis.

Conclusions: DNA hypomethylation promotes the expression of LINC00511 in breast cancer, and LINC00511 promotes the progression of breast cancer by upregulating Wnt10A, E2F2, TGFA and MET. High expression of LINC00511 is associated with poor prognosis. Our study identified the mechanism of LINC00511 upregulation and provides novel information on the progression of breast cancer.

Keywords: Breast cancer; LINC00511; DNA methylation; prognosis

Submitted Jan 20, 2021. Accepted for publication Apr 20, 2021.

doi: 10.21037 /gs-21-84

View this article at: http://dx.doi.org/10.21037/gs-21-84

$\wedge$ ORCID: 0000-0003-0368-2118. 


\section{Introduction}

Breast cancer is the most commonly diagnosed cancer in women (1). Terminal metastasis and invasion are still the primary causes of death in breast cancer patients (2). DNA methylation is the most common form of chemical modification and plays an important role in epigenetic gene regulation $(3,4)$. DNA methylation of different gene regions may lead to different biological functions and clinical effects. Some researchers have found that DNA methylation level were significantly associated to ER, PR, TP53 mutation, histological grade and mRNA subtypes, and univariate and multivariate analysis showed that the survival rate of patients with BCAP31 hypomethylation was poor, while the survival rate of patients with hypermethylation of OGG1 was lower than that of patients with normal methylation level (5). There are approximately 60,000 to 100,000 lncRNA genes in the human genome, however a few of these genes have been fully studied $(6,7)$. The newly discovered long intergenic non-protein coding RNA 00511 (LINC00511) is a $2.265 \mathrm{kB} \operatorname{lncRNA}$ located on $17 \mathrm{q} 24.3$. Studies have found that LINC00511 is highly expressed in colorectal cancer and is involved in the development of oncogenic tumors (8). Study have shown that DNA hypomethylation can activated the expression of LncRNA SNHG12 and promoted temozolomide resistance in glioblastoma (9). Other studies have identified several lncRNA regulated by aberrant DNA methylation by integrating genomewide analysis to analyze differential methylation loci and LncRNA. The result shows the expression of 5 LncRNAs (HOTAIR, HOTTIP, HOXA11-AS, HOXBAS4, and HOXC-AS3) were negatively correlated with their methylation levels (10). However, the up-regulated expression and carcinogenic mechanism of LINC00511 in breast cancer remains unknown.

In this study, we found that LINC00511 expression is closely correlated with tumor size, lymph node metastasis (LNM), and molecular subtypes of breast cancer. DNA hypomethylation could promote the expression of LINC00511, and LINC00511 reduced the sensitivity of breast cancer cells to Panobinostat. Therefore, the targeted regulation of LINC00511 expression may inhibit the progression of breast cancer and enhance the sensitivity of breast cancer cells.

We present the following article in accordance with the MDAR reporting checklist (available at http://dx.doi. org/10.21037/gs-21-84).

\section{Methods}

\section{Tissue samples}

Tissue samples were collected from January 2014 to June 2014 in the Department of Breast Surgery, Harbin Medical University Cancer Hospital (HMUCH). The tissue samples were taken from female breast cancer patients with no preoperative chemoradiotherapy, distant metastasis, or history of other tumors (Table S1). All of the samples were immediately stored in liquid nitrogen and then transferred to -80 cryogenic freezer for long-term preservation, and were not subjected to re-transport or defrosting. The pathological diagnoses were made in a double-blinded manner by two senior pathologists. All procedures were performed in accordance with the ethical standards of the responsible committee on human experimentation (institutional and national) and the Helsinki Declaration (as revised in 2013). The research was reviewed and approved by the Ethics Committees of HMUCH, and all patients who participated in the experimental study provided written informed consent.

\section{Analysis of differential gene expression}

Two breast cancer microarray datasets, GSE29431 and GSE42568, were downloaded from the GEO (https:// www.ncbi.nlm.nih.gov/geo/) to analyze the differential gene expression. High expression of lncRNA was identified by VENNY (http://bioinfogp.cnb.csic.es/tools/venny/) and Gene Expression Profiling Interactive Analysis (GEPIA) (11) (http://gepia.cancer-pku.cn/index). The genome-wide expression profiles of LINC00511 were downloaded from TCGA (https://tcga-data.nci.nih.gov/) and the Cancer Cell Line Encyclopedia (CCLE) (https:// portals.broadinstitute.org/cell/about).

\section{Cell culture}

The MDA-MB-231 and MCF-7 cell lines were obtained from the Type Culture Collection of the Chinese Academy of Sciences (Shanghai, China). The T47D, MDA-MB-468, and MCF-10a cell lines were obtained from the $\mathrm{BeNa}$ Culture Collection (Beijing, China). The MDA-MB-231 cells were cultured in L-15 medium (Gibco, USA) with $10 \%$ fetal bovine serum (FBS, Gibco, USA) in an air incubator at $37^{\circ} \mathrm{C}$. The MDA-MB-468, MCF-7, and T-47D cells were cultured in DMEM (Gibco, USA) with $10 \%$ FBS at $5 \% \mathrm{CO}_{2}$ and $37{ }^{\circ} \mathrm{C}$. The MCF-10A cells were cultured in 
a MEGM kit with $100 \mathrm{ng} / \mathrm{ml}$ cholera toxin at $5 \% \mathrm{CO}_{2}$ and $37^{\circ} \mathrm{C}$.

$R N A$ preparation and quantitative real-time polymerase chain reaction ( $q R T-P C R)$

RNA was prepared using TRIzol reagent (Invitrogen, USA) according to the manufacturer's protocol. Lipofectamine 2000 (Invitrogen, USA) was used for cell transfection. FastStart Universal SYBR Green Master (ROX) (Roche, Germany) was used to detect LINC00511 expression by qRT-PCR.

\section{DNA methylation}

The DNA methylation profile was experimentally analyzed using the Illumina Infinium HumanMethylation450 Beadchip data. The DNA methylation values were quantified as beta values and were derived from the Johns Hopkins University and University of Southern California TCGA genome characterization center; these values were recorded for each array probe in each specimen via BeadStudio software. Data regarding the H3K27ac and H3 K4me3-mediated regulation of LINC00511 were derived from ENCODE $(12,13)$.

\section{Cell transfection}

Small interfering RNA (siRNA1, siRNA2) and control RNA (siRNA-NC) were utilized for the silencing of LINC00511 expression (Sigma, USA). The pcDNA3.0 vector (oe-LINC00511) and empty DNA vector (oe-NC) were constructed for the overexpression of LINC00511 (Sigma, USA). MDA-MB-231 and MCF-7 cells were seeded in six-well plates (Corning, USA) at $1 \times 10^{6}$ cells per well to generate indicated cells.

\section{Cell migration and invasion assays}

Indicated cells in the logarithmic phase of growth were seeded in six-well plates (Corning, USA). Wounds were scratched with sterile micropipette tips, and scraped cells were washed with PBS three times. Photographs were captured under an inverted microscope. The cells $\left(5 \times 10^{5}\right)$ were seeded in Matrigel (Corning, USA)-precoated upper chambers of a 24-well Transwell plate (Corning, USA) in $200 \mu \mathrm{L}$ of serum-free medium, and the lower chamber was supplemented with $600 \mu \mathrm{L}$ of $10 \%$ serum medium.

\section{Western blot}

Western blot assays were performed as previously described (14). Anti-Met (ab137654, 1:1,000), anti-Wnt10a (ab97469, 1:1,000), anti-TGF $\alpha$ (ab208156, 1:1,000), and anti-E2F2 (ab235837, 1:1,000) were purchased from Abcam. AntiGAPDH (10494-1-AP, 1:5,000) and HRP-conjugated AffiniPure goat anti-rabbit IgG $(\mathrm{H}+\mathrm{L})$ (SA00002, 1:1,000) were purchased from Proteintech Group.

\section{Statistical analysis}

GraphPad Prism 6.0 was used for statistical processing. The relationship between clinicopathological factors and LINC00511 was compared using the chi-square test. Survival was assessed using Kaplan-Meier analysis. $\mathrm{P}<0.05$ was accepted. Each experiment is repeated three times in the laboratory.

\section{Results}

\section{LINC00511 is highly expressed in breast cancer}

Hierarchical cluster analysis showed there were 43 and 86 ncRNAs upregulated in the two breast cancer microarray datasets, respectively $(\mathrm{FC}>1.5, \mathrm{P}<0.05)$ (Figure $1 A, B)$, and seven overlapping ncRNAs in the Venn diagram: WASIR1, LINC00472, LINC00960, LINC00511, MIR143HG, MIR100HG, and HCG4 (Figure 1C). LINC00511 was significantly upregulated in breast cancer in the GEPIA database (Figure 1D,E,F,G,H,I,f). We then verified this result in the breast cancer tissue by qRT-PCR, and the result indicated that LINC00511 was significantly upregulated in breast cancer $(\mathrm{P}=0.0051)$ (Figure $1 K)$. Further studies showed that LINC00511 was also upregulated in breast cancer cell lines $(\mathrm{P}<0.05)($ Figure $1 L)$.

\section{High expression of LINC00511 is associated with the clinicopathological features of breast cancer patients, and LINC00511 promotes the migration and invasion of breast cancer in vitro}

Analysis of clinicopathological data indicated that LINC00511 was significantly correlated with the LNM $(\mathrm{P}=0.003)$, tumor size $(\mathrm{P}=0.014)$, and molecular subtypes $(\mathrm{P}=0.003)$ of breast cancer, while LINC00511 expression was not correlated with age $(\mathrm{P}=0.537)$ or TNM staging (I \& II and III \& IV) ( $\mathrm{P}=0.107)$ (Table 1).

The relative expression level of LINC00511 in the breast cancer cell lines indicated that LINC00511 was 


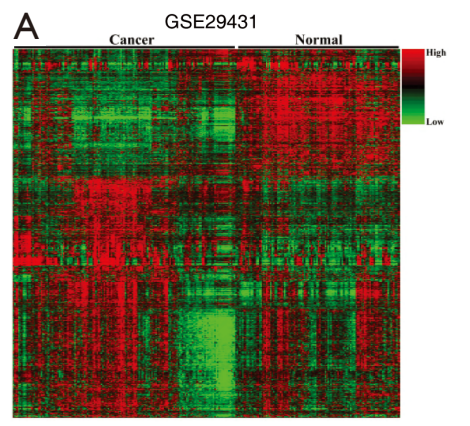

B $\underset{\text { Normal }}{\text { GSE42568 }}$

C
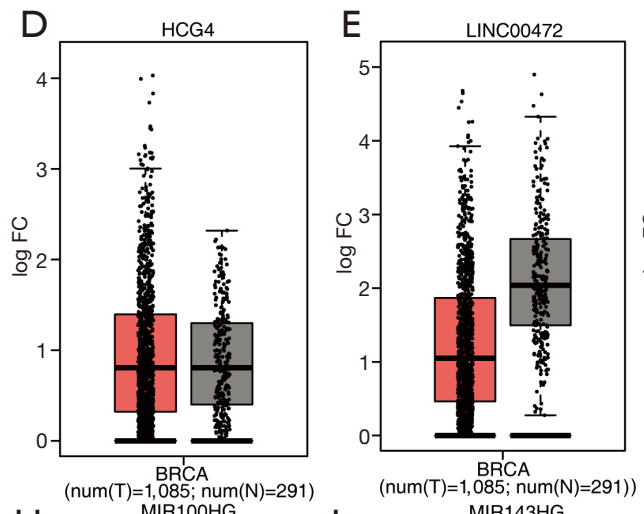

F LINC00511

G

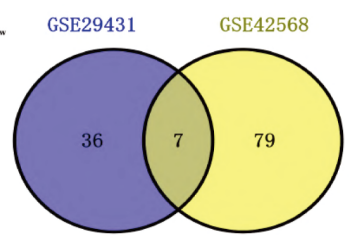

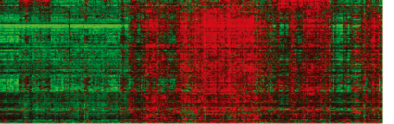
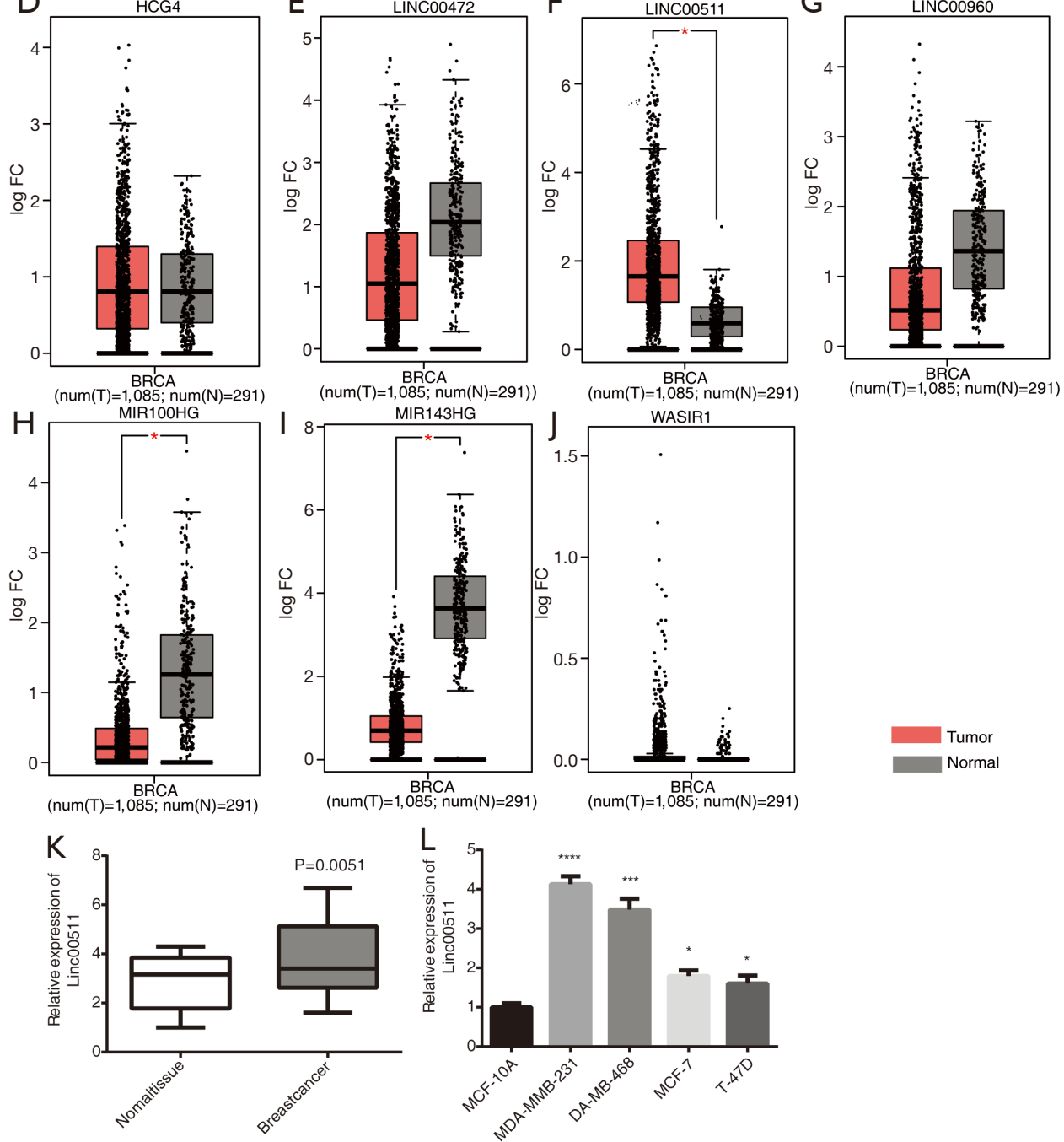

Figure 1 LINC00511 was highly expressed in breast cancer. (A,B) Hierarchical cluster analysis of differential gene expression between cancer and normal tissues in the two breast cancer microarray datasets ( $\mathrm{FC}>1.5, \mathrm{P}<0.05$, the red to green color gradient indicates the level of gene expression from high to low). (C) Overlapping ncRNAs with upregulated in the breast cancer cohorts GSE29431 and GSE42568 by VENNY (http://bioinfogp.cnb.csic.es/tools/venny/). (D,E,F,G,H,I,J) Seven overlapping ncRNAs were screened using the GEPIA database (http://gepia.cancer-pku.cn/index). (K) Expression of LINC00511 validated in breast cancer and paired normal tissues by qRT-PCR (N=96). (L) Upregulated expression of LINC00511 in breast cancer cell lines validated by qRT-PCR. *, $\mathrm{P}<0.05 ;{ }^{* *}, \mathrm{P}<0.01$; ***, $\mathrm{P}<0.001$; ****, $\mathrm{P}<0.0001$. LINC00511, long intergenic non-protein coding ribonucleic acid 00511; ncRNA, non-coding ribonucleic acid; FC, fold change; GEPIA, Gene Expression Profiling Interactive Analysis; qRT-PCR, quantitative reverse transcription polymerase chain reaction. 
Table 1 Relationship between LINC00511 expression and clinicopathological features in breast cancer. Based on the median level of LINC00511 expression, patients were divided into a high expression group ( $\mathrm{N}=48)$ and a low expression group $(\mathrm{N}=48)$

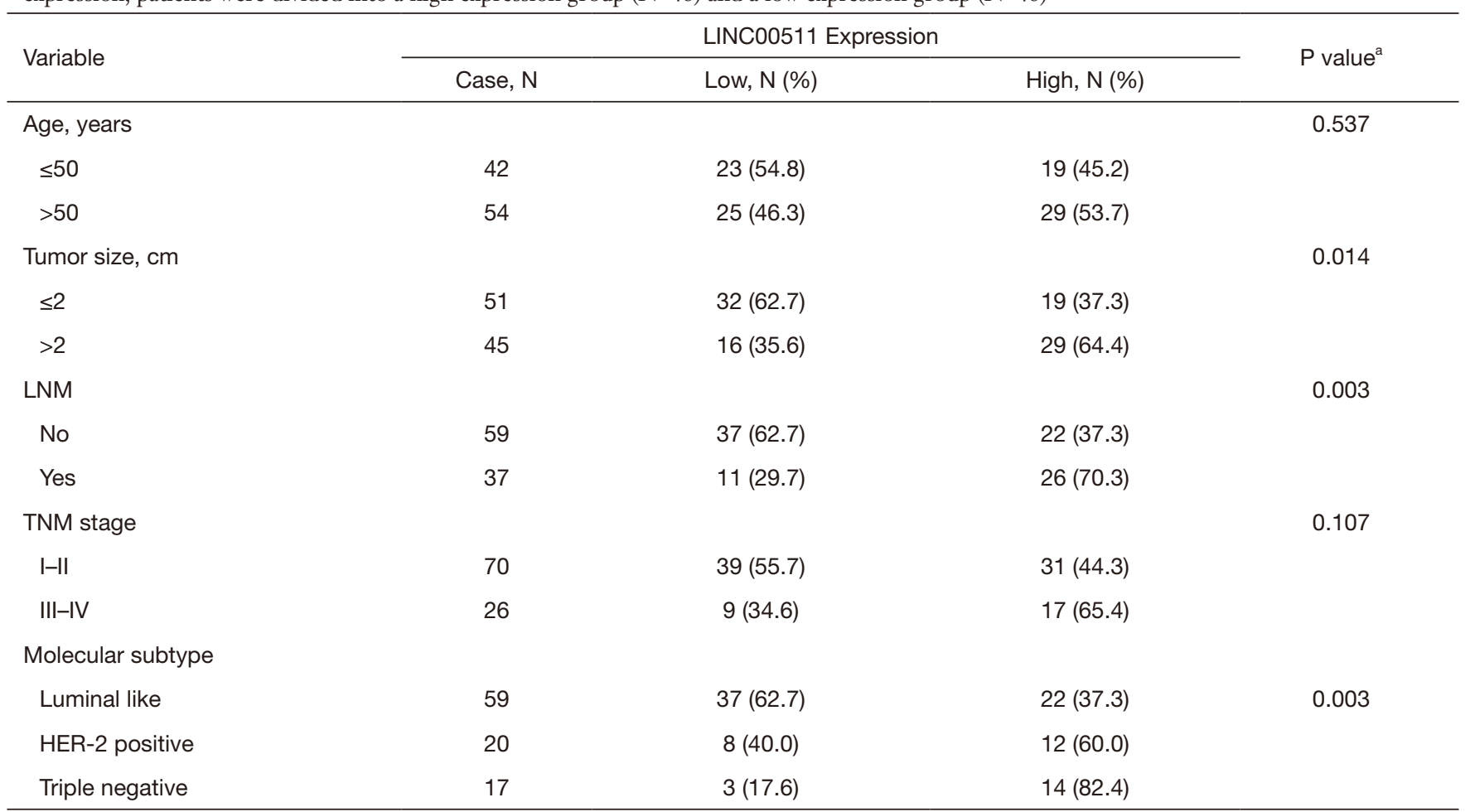

${ }^{a}, \chi^{2}$ test. $\mathrm{P}<0.05$ represents a significant difference. LNM, lymph node metastasis; TNM, tumor, node, metastasis.

highly expressed in the MDA-MB-231 and MCF7 cell lines $(\mathrm{P}<0.05)$ (Figure 1I). Moreover, LINC00511 was downregulated in the MDA-MB-231 cell lines transfected with siRNA1-LINC00511 and siRNA2-LINC00511 $(\mathrm{P}<0.05)$, and was upregulated in the MCF-7 cell line transfected with oe-LINC00511 $(\mathrm{P}<0.05)$ (Figure $2 A, B)$.

The wound healing experiment indicated that MDAMB-231 cells with silenced LINC00511 exhibited prolonged wound healing times $(\mathrm{P}<0.05)$ (Figure $2 C, D)$, and MCF-7 cells with overexpression of LINC00511 exhibited reduced wound healing times $(\mathrm{P}<0.05)$ (Figure $2 E, F)$. The results of the invasion assays also demonstrated that silenced LINC00511 expression could significantly reduce the invasion ability of the MDA-MB-231 cells $(\mathrm{P}<0.05)$ (Figure 2G,H), and that overexpression of LINC00511 could significantly promote the invasion ability of the MCF-7 cells $(\mathrm{P}<0.05)$ (Figure 2I,7).

\section{DNA bypomethylation promotes LINC00511 upregulation in breast cancer}

DNA methylation modification analysis indicated that
DNA methylation of the LINC00511 promoter locus was decreased in breast invasive carcinoma (BRCA) and 13 other types of cancer tissues (Figure $3 A$ ). Furthermore, LINC00511 expression level was found to be negatively related to DNA methylation in BRCA $(\mathrm{N}=783, \mathrm{P}<0.0001)$ (Figure $3 B$ ). Next, we also found that the DNA methylation level of the LINC00511 promoter region was lower in pancancer $(\mathrm{P}<0.0001)$ (Figure 3C), and that DNA methylation was negatively correlated with LINC00511 expression in pan-cancer $(\mathrm{N}=8451 \mathrm{P}<0.0001)$ (Figure $3 D)$. The results described above suggest that the upregulation of LINC00511 could promote the progression of breast cancer by DNA hypomethylation.

\section{LINC00511 upregulates the expression of MET, E2F2, TGFA, and WNT10A}

We identified the genes that were positively associated with LINC00511 using the Pearson correlation coefficient (PCC), and the top 200 genes were retained (PCC $\geq 0.4$, $\mathrm{P}<0.05)$. Next, gene ontology term enrichment and the Kyoto Encyclopedia of Genes and Genomes were utilized by 

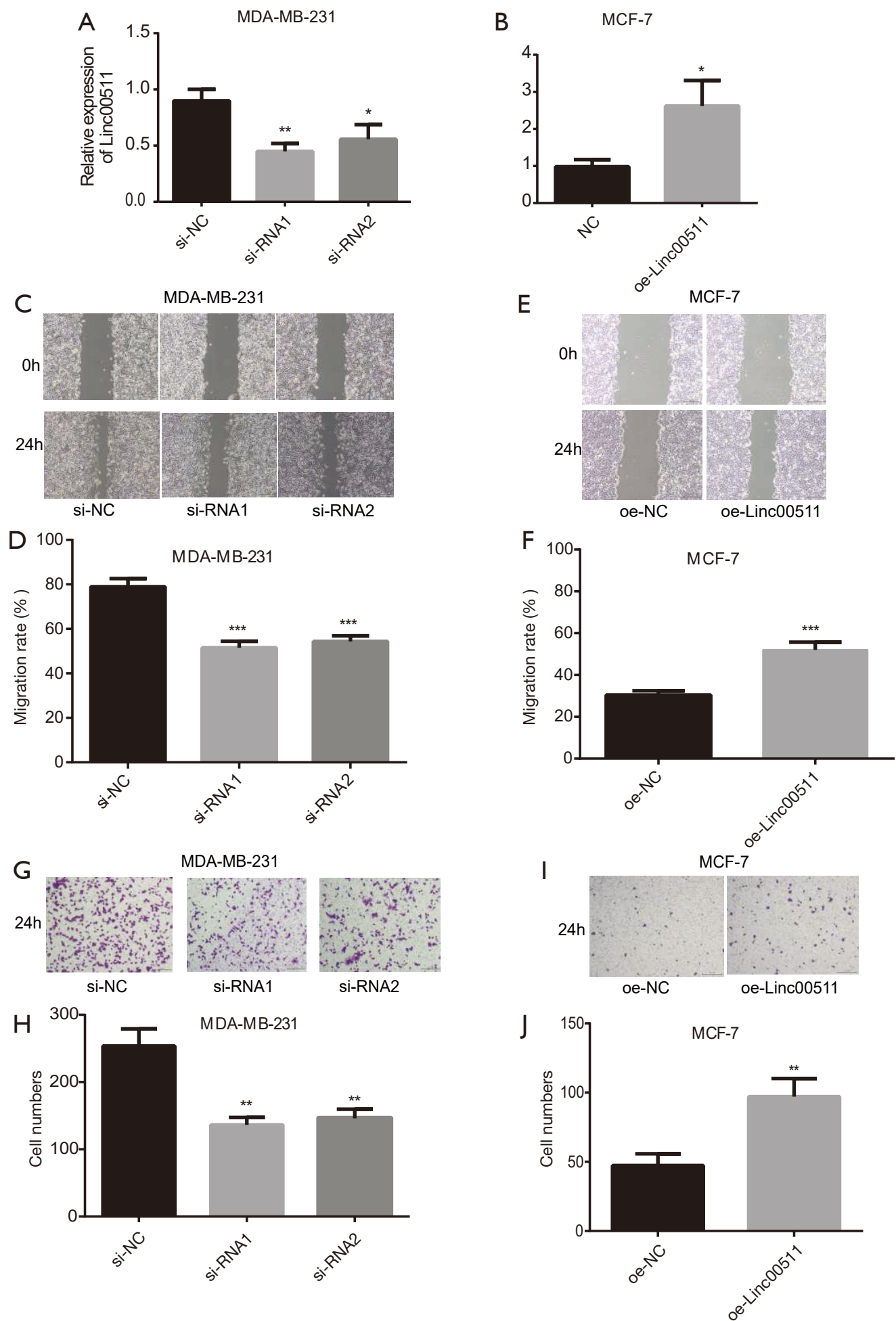

Figure 2 High expression of LINC00511 promotes the migration and invasion of breast cancer. (A,B) The expression of LINC00511 was downregulated in the MDA-MB-231 cell lines with silenced LINC00511, and was upregulated in the MCF-7 cell line with LINC00511 overexpression. (C,D,E,F) The effect of LINC00511 expression on the motility of indicated cells was detected by wound healing assays. $(\mathrm{G}, \mathrm{H}, \mathrm{I}, \mathrm{J})$ The effect of LINC00511 expression on the invasion abilities of indicated cells was detected by Matrigel invasion assays. Scale bar, $200 \mu \mathrm{m} .{ }^{*}, \mathrm{P}<0.5 ;{ }^{* *}, \mathrm{P}<0.01 ;{ }^{* * *}, \mathrm{P}<0.001 ;{ }^{* * *}, \mathrm{P}<0.0001$. 

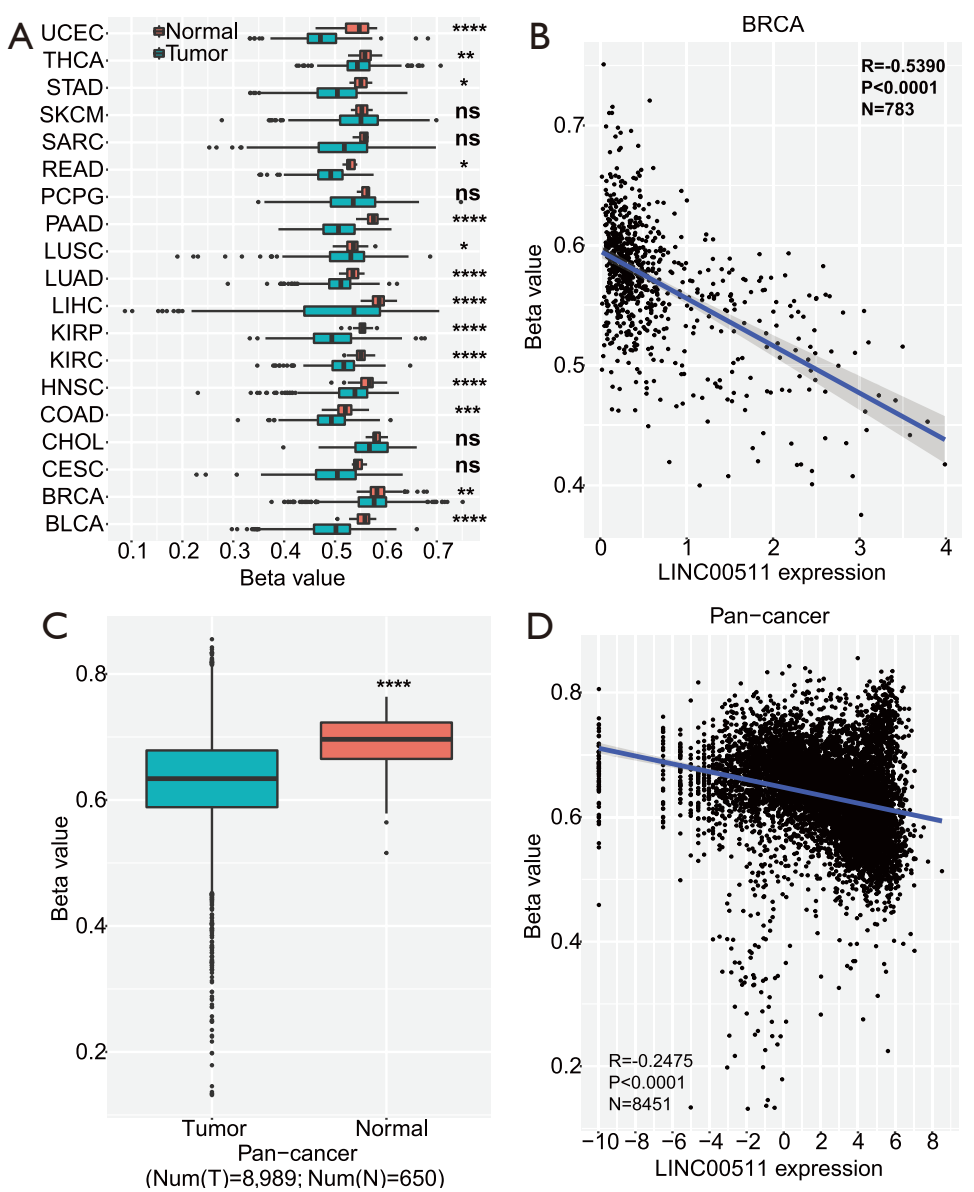

Figure 3 DNA hypomethylation promotes the upregulation of LINC00511 in breast cancer. (A) DNA methylation of the LINC00511 promoter region was decreased in 14 cancer types. (B) LINC00511 was negatively correlated with DNA methylation in BRCA. (C) DNA methylation of the LINC00511 promoter region in pan-cancer tissues was lower compared to normal tissues (T=8,989) in TCGA (https:// tcga-data.nci.nih.gov/). (D) LINC00511 was negatively correlated with DNA methylation in pan-cancer $(\mathrm{N}=8,451)$. ****, $<<0.0001$. BLCA, bladder urothelial carcinoma; BRCA, breast invasive carcinoma; CESC, cervical squamous cell carcinoma and endocervical adenocarcinoma; CHOL, Cholangio carcinoma; COAD, colon adenocarcinoma; HNSC, head and neck squamous cell carcinoma; KIRC, kidney renal clear cell carcinoma; KIRP, kidney renal papillary cell carcinoma; LIHC, liver hepatocellular carcinoma; LUAD, lung adenocarcinoma; LUSC, lung squamous cell carcinoma; PAAD, pancreatic adenocarcinoma; PCPG, pheochromocytoma and paraganglioma; READ, rectum adenocarcinoma; SARC, sarcoma; SKCM, skin cutaneous melanoma; STAD, stomach adenocarcinoma; THCA, thyroid carcinoma; UCEC, uterine corpus endometrial carcinoma; TCGA, The cancer genome atlas.

DAVID Functional Annotation Bioinformatics Microarray Analysis (https://david.ncifcrf.gov/). The results showed that LINC00511 may be associated with a variety of biological functions, including gene transcription, gene expression, intracellular signal transduction, cell proliferation, signal transduction, protein K63/K48-linked ubiquitination, cell adhesion, energy homeostasis, histone exchange, and so on (Figure 4A). In addition, LINC00511 may be involved in a variety of important oncogenic signaling pathways, such as the cell cycle, microRNAs in cancer, and natural killer cell mediated cytotoxicity (Figure 4B).

Through further verification, we found that LINC00511 was positively associated with the expression of E2F2, MET, TGFA, and WNT10A in breast cancer $(\mathrm{N}=1,104$, $\mathrm{P}<0.0001$ ) (Figure 4C,D,E,F). Our experiments also showed that the expression of E2F2, MET, TGFA, and WNT10A was downregulated when LINC00511 was silenced in the MDA-MB-231 cell line, and was upregulated when LINC00511 was overexpressed in the MCF-7 cell line (Figure $4 G$ ). 


\section{Upregulation of LINC00511 is corrected with drug sensitivity and poor prognosis in breast cancer patients}

To find the reason mentioned above, we studied the correlation between LINC00511 and Panobinostat drug sensitivity in the CCLE, and the results suggested that LINC00511 was positively correlated with the half maximal inhibitory concentration (IC50) of Panobinostat in breast cancer (Figure 5A). This indicated that LINC00511 promoted drug resistance by inhibiting the sensitivity of breast cancer cells to Panobinostat. Thus, breast cancer patients with high LINC00511 expression may have a poor prognosis.

Survival analysis showed that patients with low expression of LINC00511 had a longer overall survival (OS) time $(\mathrm{P}=0.0048)$ (Figure 5B). We then studied LINC00511 expression and OS in breast cancer patients in the TCGA database to verify our results. As we assumed, breast cancer patients with high LINC00511 had a shorter OS $(\mathrm{P}=0.0478)$ (Figure 5C). We also found that patients with high LINC00511 had a shorter OS, disease-specific survival (DSS), progression-free interval (PFI), and disease-free interval (DFI) in pan-cancer $(\mathrm{P}<0.0001)$ (Figure $5 D, E, F, G)$.

\section{Discussion}

Breast cancer has become a worldwide threat to women's health (15). Recently, many lncRNAs have been reported to be associated with progression of breast cancer. For instance, lncRNA BCRT1 is upregulated in breast cancer, and upregulates the target gene PTBP3 by binding to miR1303 (16). Although many lncRNAs have been identified in breast cancer, their functional mechanism remains unknown.

Multiple-gene sequencing can increased the detection rate of pathogenic mutant genes (17), and genome-wide association study can better find breast cancer susceptibility genes and provide more information for the development of breast cancer subtype-specific polygenic risk scores (18). In this study, we found that LINC00511 is highly expressed in breast cancer by screening differentially expressed genes in breast cancer microarray. LINC00511 is a newly discovered IncRNA in pancreatic cancer (19). It has been reported that LINC00511 is upregulated in colorectal cancer and cervical cancer $(8,20)$. Other research groups in our lab found that LINC00511 is a specific lncRNA in triple negative breast cancer (21). In our study, the expression of LINC00511 was significantly related to LNM, molecular subtype, and tumor size. However, LINC00511 was not associated with age or TNM stage. The results of bioinformatics analysis also showed that the LINC00511 was significantly correlated with the molecular typing of breast cancer, and the expression of LINC00511 was significant in triple negative breast cancer $(\mathrm{P}<0.0001$, Figure $\mathrm{S} 1 \mathrm{~A})$. And we found a negative correlation between the expression of ER (Estrogen Receptor) and LINC00511 in breast cancer $(\mathrm{P}<0.05$, Figure $\mathrm{S} 1 \mathrm{~B})$ and a positive correlation between ER and LINC00511 in normal tissues $(\mathrm{P}<0.05$, Figure $\mathrm{S} 1 \mathrm{C})$ in GEPIA database. ER change the mRNA and protein expression of DNA methyltransferase (DNMT) by recruiting recruitment co-repressors (HDAC1, histone deacetylase. PRC2, polycomb complex 2) and different protein complexes (PRC2. NurD, Nucleosome Remodeling Deacetylase) to promote DNA methylation in the promoter or enhancer region (22). ER deficiency enhance the transcriptional activity of LING00511 (23). However, Lu et al. (24) reported that the expression of LINC00511 was correlated with the TNM stage of breast cancer, but not with molecular subtypes. A possible explanation for this may be that there were no patients with stage IV breast cancer in our study, or that there were fewer patients enrolled in this study.

DNA methylation is an important epigenetic modification. Studies have found that DNA methylation is associated with the occurrence and development of breast cancer. Yang et al. (25) established a statistical prediction model based on DNA methylation data and genetic data to identify $450 \mathrm{CpG}$ islands significantly associated with the occurrence of breast cancer, of which 38 CpGs may affect breast cancer by regulating the expression of 21 genes. Teschendorff (26) analyzed the changes of DNA methylation levels in breast tissues of 50 cancer-free women and 84 paired cancers and normal tissues through a cohort study, and found that there were DNA methylation changes associated with tumorigenesis in the adjacent normal tissues of breast cancer, and $30 \%$ of the changes were increased in paired cancer tissue samples. Moreover, compared with the normal tissue on the opposite side of the tumor, the hypomethylation locus in the normal tissue on the ipsilateral side of the tumor was rich in transcription factor binding sites related to breast cancer $(\mathrm{P}<0.01)$, and the hypermethylation locus in the normal tissue on the ipsilateral side of the tumor relative to the normal tissue on the contralateral side of the tumor was depleted for $\mathrm{CpG}$ island, but enriched for adjacent shore regions $(\mathrm{P}<0.01)(27)$. Studies have found that detection of DNA methylation in peripheral blood is helpful for breast cancer risk assessment. 
A

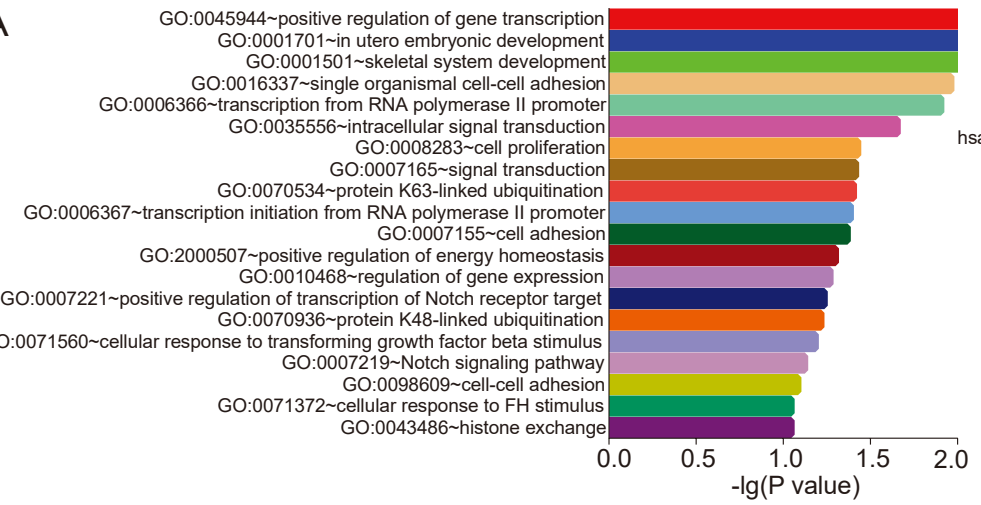

C

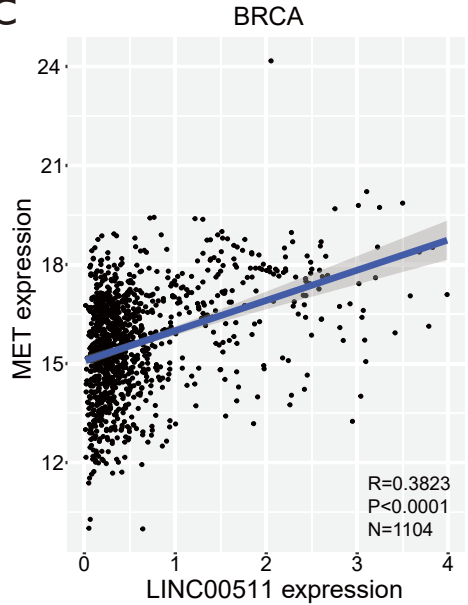

$\mathrm{F}$

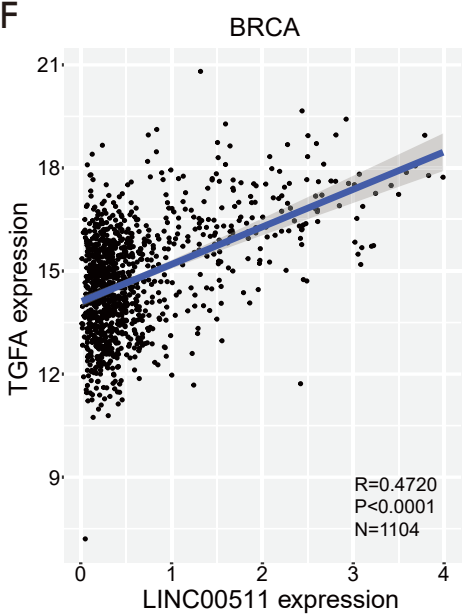

B

hsa05214:Glioma

hsa05206:MicroRNAs in cancer hsa05223:Non-small cell lung cancer hsa05212:Pancreatic cancer hsa04650:Natural killer cell mediated cytotoxicity hsa05218:Melanoma hsa05220:Chronic myeloid leukemia hsa04510:Focal adhesion hsa05222:Small cell lung cancer hsa04512:ECM-receptor interaction hsa04974:Protein digestion and absorption hsa05219:Bladder cancer hsa05200:Pathways in cancer hsa04151:PI3K-Akt signaling pathway hsa04110:Cell cycle \begin{tabular}{lll}
\hline 0 & 1 & 2 \\
& $-\lg (P$ value $)$ & 3
\end{tabular}

E
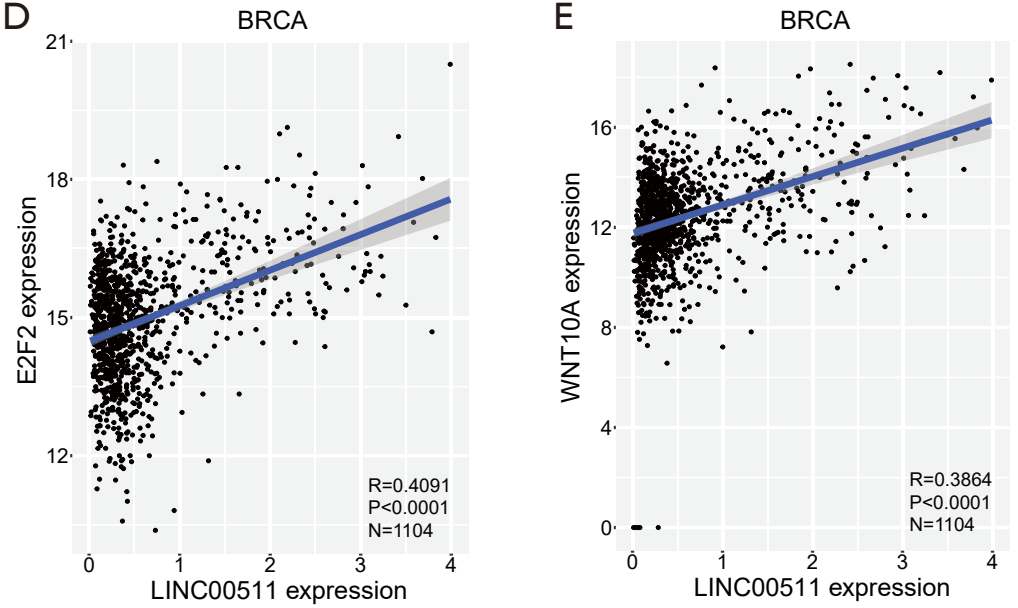

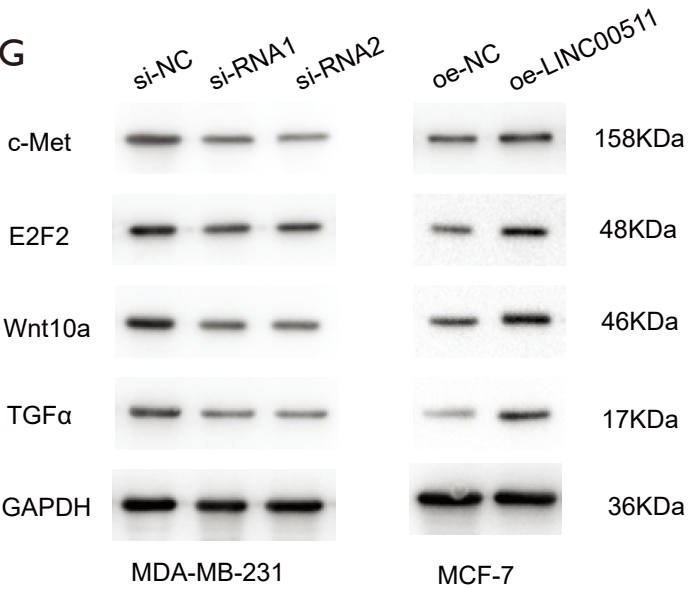

Figure 4 Predicted biological functions and pathways of LINC00511 and LINC00511 upregulate the expression of MET, E2F2, WNT10A, and TGFA. (A) The potential biological function of LINC00511 in breast cancer. (B) The potential carcinogenic pathway of LINC00511 in breast cancer. (C,D,E,F) The expression of LINC00511 was positively correlated with the expression of MET, E2F2, TGFA, and WNT10A in TCGA (N=1,104). (G) LINC00511 promoted the expression of MET, E2F2, TGFA, and WNT10A in breast cancer cell lines by western blot. TCGA, the cancer genome atlas. 
A
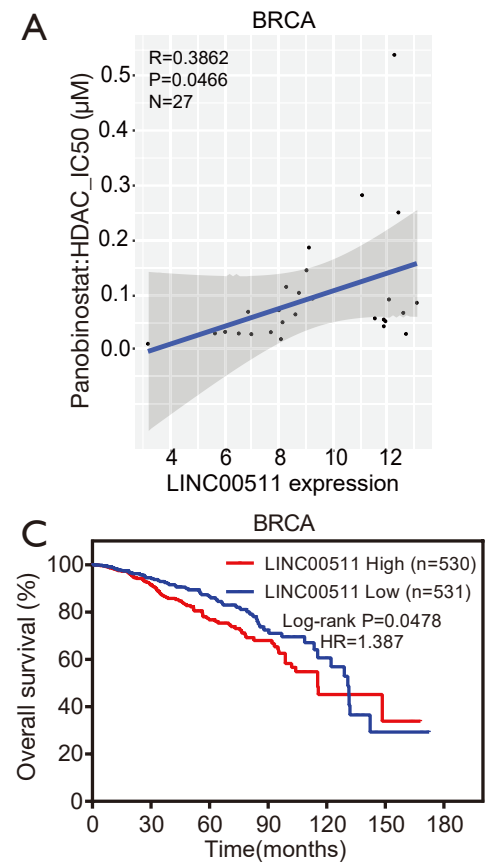

$\mathrm{E}$

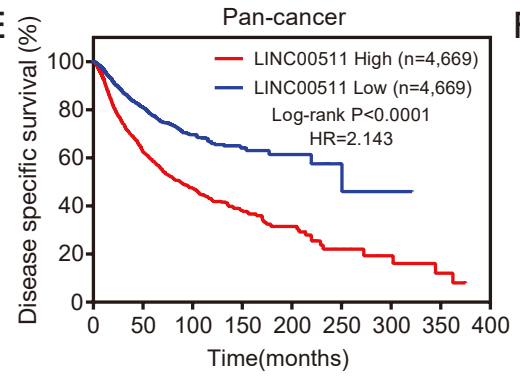

G

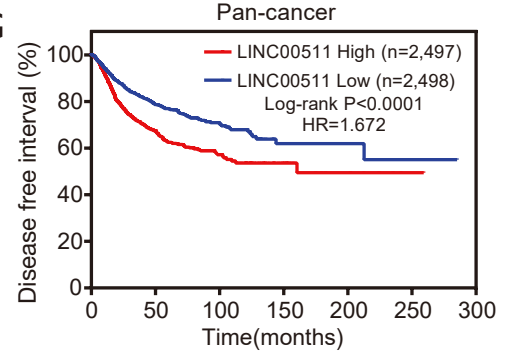

B
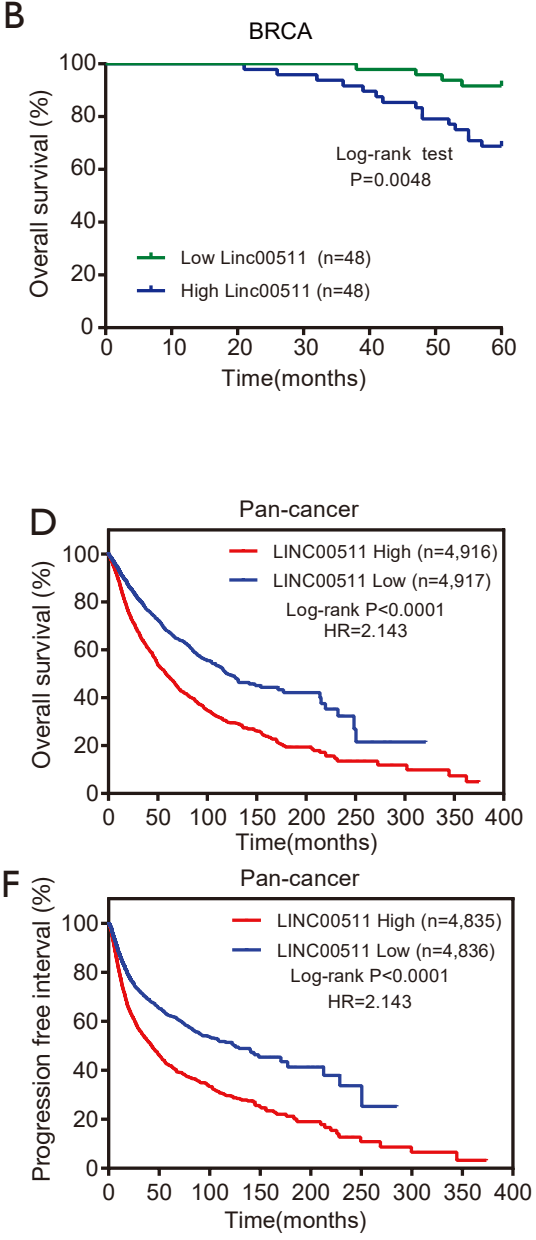

Figure 5 High expression of LINC00511 is corrected with drug resistance and prognosis in breast cancer. (A) LINC00511 was positively related to the half maximal inhibitory concentration (IC50) of Panobinostat: HDAC (N=27) in breast cancer cells. (B) Breast cancer patients with low LINC00511 expression had a longer OS in HMUCH. (C) Breast cancer patients with low LINC00511 had a longer OS in TCGA. (D,E,F,G) TCGA data showed that high LINC00511 expression resulted in worse OS, DSS, PFI, and DFI in pan-cancer. HDAC, histone deacetylase; OS, overall survival; DSS, disease specific survival; PFI, progression free interval; DFI, disease free interval; HMUCH, Harbin Medical University Cancer Hospital; TCGA, the cancer genome atlas.

Through the analysis of genomic DNA methylation in peripheral blood DNA samples of breast cancer patients, some researchers have found a heritable DNA methylation marker that can be used to identify the risk of breast cancer, the coding gene is: GREB1, PNKD, C7orf50, TMC3 (28). A multicenter study found that blood-based whole-genome bisulfite sequencing can improve the accuracy of noninvasive diagnostic imaging of early breast cancer (29). 
However, the mechanism through which upregulated LINC00511 expression promotes breast cancer remains unknown. Studies have found that lncRNA expression is related to the regulation of DNA methylation $(9,30)$, and we speculated that LINC00511 expression might be regulated by DNA methylation. In our study, we found that the DNA methylation of the LINC00511 promoter region was decreased in breast cancer, and DNA hypomethylation was significantly positively correlated with LINC00511 expression, indicating that DNA hypomethylation promotes LINC00511 expression.

In addition, our study found that high expression of LINC00511 could promote the migration and invasion of breast cancer cells. Moreover, LINC00511 performs a variety of potential biological functions, including cell proliferation, regulation of gene expression, and so on. And LINC00511 may promote the malignant behavior of breast cancer via the PI3K-Akt signaling pathway, focal adhesion, and other pathways. Our assays showed that the expression of MET, TGFA, E2F2, and WNT10A was downregulated when LINC00511 was silenced in the MDA-MB-231 cell line, and was upregulated when LINC00511 was overexpressed in the MCF-7 breast cancer cell line. These results indicate that LINC00511 promoted the expression of key genes, such as Wnt10A, E2F2, TGFA, and MET, which participate in breast cancer progression. It has been previously reported that LINC00511 can be used as a scaffold by binding to EZH2, which can inhibit the expression of target gene p57 to promote the progression of breast cancer (31). Moreover, LINC00511 can also be used as a molecular stent to promote angiogenesis in pancreatic cancer by binding to miRNA to eliminate its effect (19).

Panobinostat is a histone deacetylase inhibitor that is used in the treatment of multiple myelomas (32). Studies have reported that Panobinostat has a good antitumor effect in breast cancer (33). However, in our study, LINC00511 inhibited the sensitivity of breast cancer to Panobinostat, suggesting that it may give rise to drug resistance in breast cancer, and thus, the prognosis for breast cancer patients with high LINC00511 expression is poor. Our study found that DNA methylation enhances breast cancer resistance by promoting the expression of LINC00511. It has been found that cancer cells show therapy resistance by changing the DNA methylation, DNA methylation modified tumor suppressor gene hypermethylation associated with chemotherapy resistance in cancer: TGBI, ER-a, MSH2, MGP, PSAT1 and hypomethylation associated with chemotherapy resistance in cancer: ID4, ERp29/MGMT, ETS-1, miR-663, MDR1 (34). Next, we evaluated the association between LINC00511 and OS of breast cancer patients by KaplanMeier analysis. The results indicated that breast cancer patients with upregulated LINC00511 had a shorter OS. We also verified this result in TCGA database. Moreover, pan-cancer patients with high LINC00511 had a poor prognosis in TCGA. This study validated our hypothesis that breast cancer patients with high expression of LINC00511 have a poor prognosis.

NSUN2 is an RNA methyltransferase. The researchers found that DNA hypomethylation promotes the expression of NSUN2 in breast cancer, and the overexpression of NSUN2 promotes the migration and invasion of breast cancer cells (35). Nuclear transport protein $\alpha 2$ is a nuclear import factor, KPNA2 is associated with poor prognosis of breast cancer (36). It has been found that silencing the expression of KPNA2 can inhibit the proliferation, migration, invasion and cell cycle arrest of breast cancer cells (37). It was found that the expression of LINC00511 was significantly correlated with NSUN2 and KPNA2 (Figure S1D,E). The limitation of this paper is that there is no more in-depth study on the mechanism of action between NSUN2 and KPNA2 and DNA hypomethylation.

\section{Conclusions}

LINC00511 was upregulated in human breast cancer. Additionally, breast cancer patients with upregulated LINC00511 expression have a poor prognosis. DNA hypomethylation of the LINC00511 promoter region promotes the upregulation of LINC00511 expression, and LINC00511 contributes to breast cancer progression by promoting the expression of MET, TGFA, E2F2, and WNT10A and inhibiting the sensitivity of breast cancer cells to Panobinostat.

\section{Acknowledgments}

We thank Harbin Medical University Cancer Hospital and Translational Medicine Research and Cooperation Center of Northern China for their technical support.

Funding: This work was supported by funding from the National Natural Science Foundation of China (Grant Number: 81372837) and the Scientific Research Project of Education Department of Heilongjiang Province (Grant Number: 1253HQ002). 


\section{Footnote}

Reporting Checklist: The authors have completed the MDAR reporting checklist. Available at http://dx.doi.org/10.21037/ gs-21-84

Data Sharing Statement: Available at http://dx.doi. org/10.21037/gs-21-84

Conflicts of Interest: All authors have completed the ICMJE uniform disclosure form (available at http://dx.doi. org/10.21037/gs-21-84). The authors have no conflicts of interest to declare.

Ethical Statement: The authors are accountable for all aspects of the work in ensuring that questions related to the accuracy or integrity of any part of the work are appropriately investigated and resolved. All procedures were performed in accordance with the ethical standards of the responsible committee on human experimentation (institutional and national) and the Helsinki Declaration (as revised in 2013). This study was approved by the research ethics committee of Harbin Medical University Cancer Hospital and informed consent was taken from all individual participants.

Open Access Statement: This is an Open Access article distributed in accordance with the Creative Commons Attribution-NonCommercial-NoDerivs 4.0 International License (CC BY-NC-ND 4.0), which permits the noncommercial replication and distribution of the article with the strict proviso that no changes or edits are made and the original work is properly cited (including links to both the formal publication through the relevant DOI and the license). See: https://creativecommons.org/licenses/by-nc-nd/4.0/.

\section{References}

1. Bray F, Ferlay J, Soerjomataram I, et al. Global cancer statistics 2018: GLOBOCAN estimates of incidence and mortality worldwide for 36 cancers in 185 countries. CA Cancer J Clin 2018;68:394-424.

2. Siegel RL, Miller KD, Jemal A. Cancer statistics, 2020. CA Cancer J Clin 2020;70:7-30.

3. Cavalli G, Heard E. Advances in epigenetics link genetics to the environment and disease. Nature 2019;571:489-99.

4. Jung G, Hernandez-Illan E, Moreira L, et al. Epigenetics of colorectal cancer: biomarker and therapeutic potential.
Nat Rev Gastroenterol Hepatol 2020;17:111-30.

5. Fleischer T, Edvardsen H, Solvang HK, et al. Integrated analysis of high-resolution DNA methylation profiles, gene expression, germline genotypes and clinical end points in breast cancer patients. Int J Cancer 2014;134:2615-25.

6. Carlevaro-Fita J, Johnson R. Global Positioning System: Understanding Long Noncoding RNAs through Subcellular Localization. Mol Cell 2019;73:869-83.

7. Lucas T, Bonauer A, Dimmeler S. RNA Therapeutics in Cardiovascular Disease. Circ Res 2018;123:205-20.

8. Sun S, Xia C, Xu Y. HIF-1alpha induced lncRNA LINC00511 accelerates the colorectal cancer proliferation through positive feedback loop. Biomed Pharmacother 2020;125:110014.

9. Lu C, Wei Y, Wang X, et al. DNA-methylation-mediated activating of lncRNA SNHG12 promotes temozolomide resistance in glioblastoma. Mol Cancer 2020;19:28.

10. Song P, Wu L, Guan W. Genome-Wide Identification and Characterization of DNA Methylation and Long NonCoding RNA Expression in Gastric Cancer. Front Genet 2020;11:91.

11. Tang Z, Li C, Kang B, et al. GEPIA: a web server for cancer and normal gene expression profiling and interactive analyses. Nucleic Acids Res 2017;45:W98-W102.

12. Font-Tello A, Kesten N, Xie Y, et al. FiTAc-seq: fixedtissue ChIP-seq for H3K27ac profiling and super-enhancer analysis of FFPE tissues. Nat Protoc 2020;15:2503-18.

13. Leng $X$, Wang J, An N, et al. Histone 3 lysine-27 demethylase KDM6A coordinates with KMT2B to play an oncogenic role in NSCLC by regulating H3K4me3. Oncogene 2020;39:6468-79.

14. Ma F, Liu X, Zhou S, et al. Long non-coding RNA FGF13-AS1 inhibits glycolysis and stemness properties of breast cancer cells through FGF13-AS1/IGF2BPs/Myc feedback loop. Cancer Lett 2019;450:63-75.

15. DeSantis CE, Ma J, Gaudet MM, et al. Breast cancer statistics, 2019. CA Cancer J Clin 2019;69:438-51.

16. Liang Y, Song X, Li Y, et al. LncRNA BCRT1 promotes breast cancer progression by targeting miR-1303/PTBP3 axis. Mol Cancer 2020;19:85.

17. Kurian AW, Ward KC, Hamilton AS, et al. Uptake, Results, and Outcomes of Germline Multiple-Gene Sequencing After Diagnosis of Breast Cancer. JAMA Oncol 2018;4:1066-72.

18. Zhang H, Ahearn TU, Lecarpentier J, et al. Genomewide association study identifies 32 novel breast cancer susceptibility loci from overall and subtype-specific analyses. Nat Genet 2020;52:572-81. 
19. Zhao X, Liu Y, Li Z, et al. Linc00511 acts as a competing endogenous RNA to regulate VEGFA expression through sponging hsa-miR-29b-3p in pancreatic ductal adenocarcinoma. J Cell Mol Med 2018;22:655-67.

20. Shi Y, Liu M, Huang Y, et al. Promotion of cell autophagy and apoptosis in cervical cancer by inhibition of long noncoding RNA LINC00511 via transcription factor RXRA-regulated PLD1. J Cell Physiol 2020;235:6592-604.

21. Xu S, Kong D, Chen Q, et al. Oncogenic long noncoding RNA landscape in breast cancer. Mol Cancer 2017;16:129.

22. Kovacs T, Szabo-Meleg E, Abraham IM. Estradiol-Induced Epigenetically Mediated Mechanisms and Regulation of Gene Expression. Int J Mol Sci 2020;21:3177.

23. Zhang J, Sui S, Wu H, et al. The transcriptional landscape of lncRNAs reveals the oncogenic function of LINC00511 in ER-negative breast cancer. Cell Death Dis 2019;10:599.

24. Lu G, Li Y, Ma Y, et al. Long noncoding RNA LINC00511 contributes to breast cancer tumourigenesis and stemness by inducing the miR-185-3p/E2F1/Nanog axis. J Exp Clin Cancer Res 2018;37:289.

25. Yang Y, Wu L, Shu XO, et al. Genetically Predicted Levels of DNA Methylation Biomarkers and Breast Cancer Risk: Data From 228951 Women of European Descent. J Natl Cancer Inst 2020;112:295-304.

26. Teschendorff AE, Gao Y, Jones A, et al. DNA methylation outliers in normal breast tissue identify field defects that are enriched in cancer. Nat Commun 2016;7:10478.

27. Muse ME, Titus AJ, Salas LA, et al. Enrichment of CpG island shore region hypermethylation in epigenetic breast field cancerization. Epigenetics 2020;15:1093-106.

28. Joo JE, Dowty JG, Milne RL, et al. Heritable DNA methylation marks associated with susceptibility to breast cancer. Nat Commun 2018;9:867.

29. Liu J, Zhao H, Huang Y, et al. Genome-wide cell-free DNA methylation analyses improve accuracy of noninvasive diagnostic imaging for early-stage breast cancer.

Cite this article as: Liu C, Xu Y, Liu X, Fu Y, Zhu K, Niu Z, Liu J, Qian C. Upregulation of LINC00511 expression by DNA hypomethylation promotes the progression of breast cancer. Gland Surg 2021;10(4):1418-1430. doi: 10.21037/gs-21-84
Mol Cancer 2021;20:36.

30. Kim-Wanner SZ, Assenov Y, Nair MB, et al. GenomeWide DNA Methylation Profiling in Early Stage I Lung Adenocarcinoma Reveals Predictive Aberrant Methylation in the Promoter Region of the Long Noncoding RNA PLUT: An Exploratory Study. J Thorac Oncol 2020;15:1338-50.

31. Sun CC, Li SJ, Li G, et al. Long Intergenic Noncoding RNA 00511 Acts as an Oncogene in Non-small-cell Lung Cancer by Binding to EZH2 and Suppressing p57. Mol Ther Nucleic Acids 2016;5:e385.

32. Hall CJ, Doss S, Hay N, et al. NICE guidance on panobinostat for patients with multiple myeloma after at least two previous treatments. Lancet Oncol 2016;17:279-80.

33. Fedele P, Orlando L, Cinieri S. Targeting triple negative breast cancer with histone deacetylase inhibitors. Expert Opin Investig Drugs 2017;26:1199-206.

34. Romero-Garcia S, Prado-Garcia H, Carlos-Reyes A. Role of DNA Methylation in the Resistance to Therapy in Solid Tumors. Front Oncol 2020;10:1152.

35. Yi J, Gao R, Chen Y, et al. Overexpression of NSUN2 by DNA hypomethylation is associated with metastatic progression in human breast cancer. Oncotarget 2017;8:20751-65.

36. Alshareeda AT, Negm OH, Green AR, et al. KPNA2 is a nuclear export protein that contributes to aberrant localisation of key proteins and poor prognosis of breast cancer. Br J Cancer 2015;112:1929-37.

37. Duan M, Hu F, Li D, et al. Silencing KPNA2 inhibits IL6-induced breast cancer exacerbation by blocking NFkappaB signaling and c-Myc nuclear translocation in vitro. Life Sci 2020;253:117736.

(English Language Editor: A. Kassem) 


\section{Supplementary}

Table S1 Age and sex for all study participants

\begin{tabular}{|c|c|c|}
\hline Serial number & Sex & Age \\
\hline 1 & Female & 47 \\
\hline 2 & Female & 48 \\
\hline 3 & Female & 62 \\
\hline 4 & Female & 46 \\
\hline 5 & Female & 53 \\
\hline 6 & Female & 64 \\
\hline 7 & Female & 59 \\
\hline 8 & Female & 39 \\
\hline 9 & Female & 70 \\
\hline 10 & Female & 46 \\
\hline 11 & Female & 46 \\
\hline 12 & Female & 61 \\
\hline 13 & Female & 78 \\
\hline 14 & Female & 54 \\
\hline 15 & Female & 38 \\
\hline 16 & Female & 33 \\
\hline 17 & Female & 57 \\
\hline 18 & Female & 42 \\
\hline 19 & Female & 52 \\
\hline 22 & Female & 44 \\
\hline 20 & Female & 49 \\
\hline 21 & Female & 73 \\
\hline 23 & Female & 56 \\
\hline 24 & Female & 56 \\
\hline 25 & Female & 48 \\
\hline 26 & Female & 46 \\
\hline 27 & Female & 51 \\
\hline 28 & Female & 55 \\
\hline 29 & Female & 36 \\
\hline 30 & Female & 48 \\
\hline 31 & Female & 60 \\
\hline 32 & Female & 48 \\
\hline 33 & Female & 60 \\
\hline 34 & Female & 55 \\
\hline 35 & Female & 61 \\
\hline
\end{tabular}

Table S1 (continued)
Table S1 (continued)

\begin{tabular}{|c|c|c|}
\hline Serial number & Sex & Age \\
\hline 36 & Female & 63 \\
\hline 37 & Female & 63 \\
\hline 38 & Female & 40 \\
\hline 39 & Female & 43 \\
\hline 40 & Female & 46 \\
\hline 41 & Female & 65 \\
\hline 42 & Female & 32 \\
\hline 43 & Female & 44 \\
\hline 44 & Female & 66 \\
\hline 45 & Female & 56 \\
\hline 46 & Female & 64 \\
\hline 47 & Female & 59 \\
\hline 48 & Female & 55 \\
\hline 49 & Female & 48 \\
\hline 50 & Female & 56 \\
\hline 51 & Female & 32 \\
\hline 52 & Female & 66 \\
\hline 53 & Female & 46 \\
\hline 54 & Female & 49 \\
\hline 55 & Female & 47 \\
\hline 56 & Female & 38 \\
\hline 57 & Female & 46 \\
\hline 58 & Female & 56 \\
\hline 59 & Female & 44 \\
\hline 60 & Female & 53 \\
\hline 61 & Female & 44 \\
\hline 62 & Female & 58 \\
\hline 63 & Female & 51 \\
\hline 64 & Female & 44 \\
\hline 65 & Female & 64 \\
\hline 66 & Female & 59 \\
\hline 67 & Female & 46 \\
\hline 68 & Female & 63 \\
\hline 69 & Female & 69 \\
\hline 70 & Female & 50 \\
\hline
\end{tabular}

Table S1 (continued) 
Table S1 (continued)

\begin{tabular}{|c|c|c|}
\hline Serial number & Sex & Age \\
\hline 71 & Female & 61 \\
\hline 72 & Female & 46 \\
\hline 73 & Female & 41 \\
\hline 74 & Female & 64 \\
\hline 75 & Female & 55 \\
\hline 76 & Female & 50 \\
\hline 77 & Female & 61 \\
\hline 78 & Female & 59 \\
\hline 79 & Female & 50 \\
\hline 80 & Female & 63 \\
\hline 81 & Female & 40 \\
\hline 82 & Female & 46 \\
\hline 83 & Female & 52 \\
\hline 84 & Female & 73 \\
\hline 85 & Female & 63 \\
\hline 86 & Female & 68 \\
\hline 87 & Female & 64 \\
\hline 88 & Female & 53 \\
\hline 89 & Female & 61 \\
\hline 90 & Female & 39 \\
\hline 91 & Female & 61 \\
\hline 92 & Female & 59 \\
\hline 93 & Female & 53 \\
\hline 94 & Female & 42 \\
\hline 95 & Female & 45 \\
\hline 96 & Female & 62 \\
\hline
\end{tabular}


A

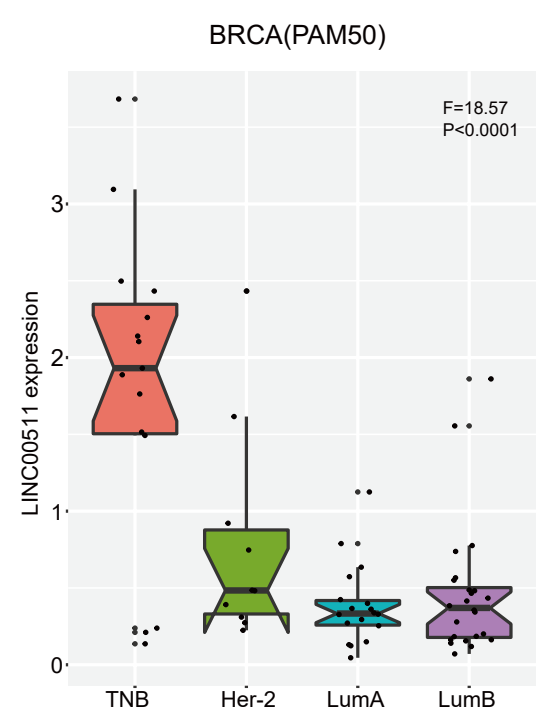

B

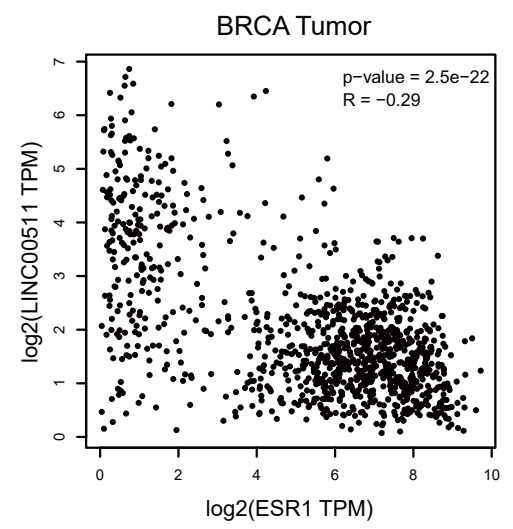

C

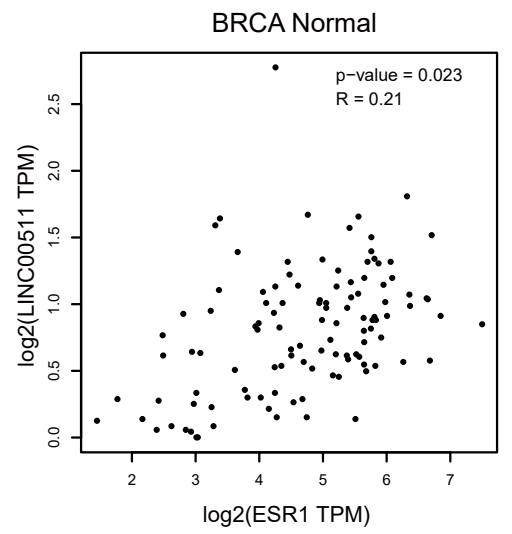

D

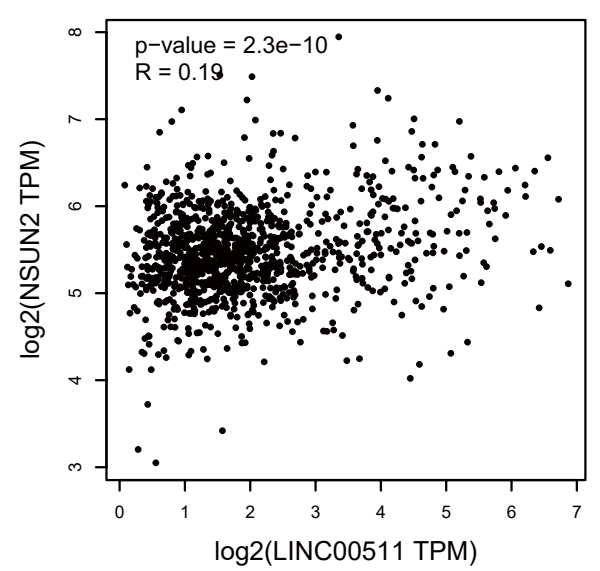

$\mathrm{E}$

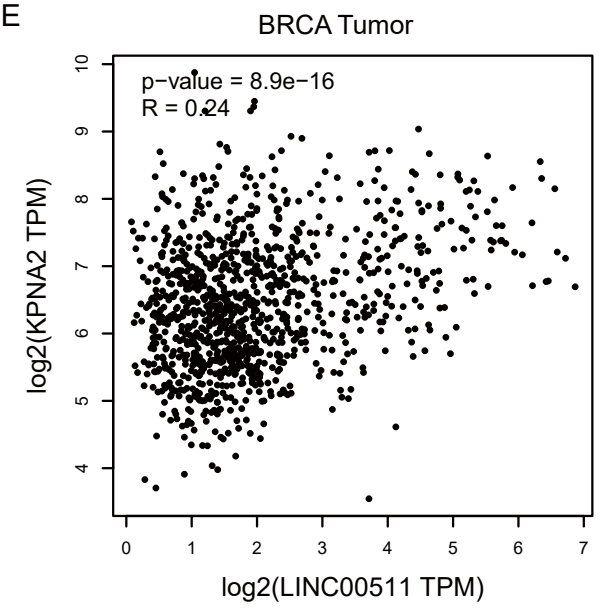

Figure S1 Analysis of the relationship between LINC00511 and molecular subtypes of breast cancer, estrogen receptor, NSUN2 and KPNA2. (A) LINC00511 was significantly correlated with the molecular typing of breast cancer in TCGA. (B) GEPIA database analysis showed that the expression of ER is negatively correlated with LINC00511 in breast cancer. (C) There is a positive correlation between the expression of estrogen receptor and LINC00511 in normal tissues adjacent to breast cancer in GEPIA. (D,E) The expression of LINC00511 was significantly correlated with NSUN2 and KPNA2 in GEPIA. P<0.05 means statistical significance. TCGA, the cancer genome atlas; ER, estrogen receptor; GEPIA, Gene Expression Profiling Interactive Analysis. 